Théologiques

Théologiques

\title{
Jésus aux yeux de la spiritualité
}

\section{Jean-Claude Breton}

Volume 18, numéro 2, 2010

Les lieux de la spiritualité aujourd'hui

URI : https://id.erudit.org/iderudit/1007481ar

DOI : https://doi.org/10.7202/1007481ar

Aller au sommaire du numéro

\section{Éditeur(s)}

Faculté de théologie et de sciences des religions, Université de Montréal

ISSN

1188-7109 (imprimé)

1492-1413 (numérique)

Découvrir la revue

Citer cet article

Breton, J.-C. (2010). Jésus aux yeux de la spiritualité. Théologiques, 18(2), 83-101. https://doi.org/10.7202/1007481ar

\section{Résumé de l'article}

Le présent article formule une opinion selon laquelle la compréhension de Jésus est fortement conditionnée par la vie spirituelle de ceux et celles qui s'intéressent à lui. Sans négliger les résultats de l'histoire, il appert bien souvent que le sens accordé à l'image de Jésus retenue dépend plus de la vie de foi et des engagements personnels que de la force des arguments scientifiques. Le Jésus réel échappera toujours en partie à la quête historique, mais il peut s'imposer aux personnes qui le découvrent avec les yeux de leur foi. 


\title{
Jésus aux yeux de la spiritualité
}

\author{
Jean-Claude BRETON* \\ Théologie et sciences des religions \\ Université de Montréal
}

Dans les milieux catholiques du début du $\mathrm{xx}^{\mathrm{e}}$ siècle, la possibilité d'écrire une vie de Jésus qui réponde aux exigences de la méthode historique avait encore cours. Penser autrement aurait sans doute été compris comme une concession au mouvement moderniste des Loisy ${ }^{1}$ et autres condamnés par l'encyclique Pascendi domici gregis (1907) de Pie X. Un Daniel-Rops (1901-1965), par exemple, passa d'écrivain prolifique à auteur populaire avec la publication de Jésus en son temps en 1945. Mais ces propos catholiques n'avaient pas pris en compte ce qui se passait du côté protestant.

Depuis le texte d'Albert Schweitzer, Recherches sur la vie de Jésus (1994 [1906]), les questions sur le Jésus historique ont pris une tournure insoupçonnable jusque-là. Dans les milieux protestants d'abord, où Rudolf Bultmann prendra la relève avec son projet de démythologisation, puis dans les milieux catholiques à mesure que les exigences de la recherche scientifique sur la Bible s'y répandront. Cette remise en question était la deuxième étape dans la quête du Jésus historique. Car il est communément accepté aujourd'hui que le débat sur le Jésus historique et le Jésus de la foi a connu trois grandes étapes, que Marguerat (2003) a bien identifiées. La première étape correspondait aux recherches historiques du XIX ${ }^{\mathrm{e}}$ siècle et elle s'achèvera avec la remise en question de Schweitzer. La troisième étape de la quête sera préparée par la réaction de Käsemann aux positions de Bultmann, mais elle se prolonge dans certains travaux comme ceux de John P. Meier (2005). Ses investigations fascinantes peuvent laisser

* Jean-Claude Breton est doyen de la Faculté de théologie et de sciences des religions de l'Université de Montréal. Il a enseigné la vie spirituelle pendant plus de vingt ans et a fait sa thèse de doctorat sur Marcel Légaut.

1. Dans son petit livre L'Évangile et l'Église, qui sera appelé livre rouge à partir de sa $2^{\mathrm{e}}$ édition, Alfred Loisy (18571940) défend des positions pour lesquelles il fut condamné en 1908, mais qui sont devenues aujourd'hui d'enseignement commun dans les facultés de théologie. 
l'impression qu'avec une telle somme d'informations, la discussion va s'estomper, mais ce serait bien mal comprendre les leçons de l'histoire.

On pourrait illustrer cette référence à l'histoire par l'exemple de ce qui s'est passé autour de Bultmann et Käsemann. On aurait pu croire en effet que les propos du premier sur la démythologisation venaient de mettre fin au questionnement et qu'il n'y aurait plus personne à vouloir rechercher le Jésus historique. Et voilà que le "disciple " publie un ouvrage, Le problème du Jésus historique (1972 [1954]), qui remet en question les positions du maître. Ce qui signifie bien à mon avis qu'au gré des découvertes et à même la diversité des opinions, on n'a pas fini de traiter de la question du Jésus historique chez les biblistes.

S'il faut se réjouir de cette vitalité et parfois de cette fécondité de la recherche biblique sur le sujet du Jésus historique, on peut aussi se demander si cette recherche ne pourrait pas s'accompagner d'un autre type de réflexion. Comme le souligne Sandra M. Schneiders dans son livre Le texte de la rencontre, les auteurs du Nouveau Testament n'ont pas écrit seulement pour offrir des objets de recherche aux biblistes des générations à venir, mais pour répondre au questionnement inscrit dans leur démarche spirituelle ${ }^{2}$. S'il est important de mettre en œuvre toutes les méthodes disponibles pour lire adéquatement les textes qu'ils nous ont laissés, on peut aussi se demander si la communion à leur quête spirituelle ne serait pas aussi instructive. Schneiders soutient encore que, même s'ils observent toutes les règles de l'art, les biblistes qui concentrent leur travail sur la Bible, plutôt que sur un autre document ancien, le font sans doute au nom de la reconnaissance plus ou moins explicite de la valeur spirituelle de ce texte.

Dans son livre La vérité s'accomplit (2009), Gagey pose la question du Jésus de l'histoire et il évoque les succès de l'enquête historique critique, pour finalement ajouter ceci :

Ainsi le procès dont son intervention dans l'histoire fit et fait encore l'objet ne se joue pas primairement sur la scrupuleuse exactitude des informations

2. Voici à ce titre, la réflexion qu'elle fait à partir de la réponse de son professeur à la question de savoir si le «fait que l'Ancien Testament soit inspiré » changeait quelque chose à sa lecture. Ce professeur de l'Institut catholique de Paris avait répondu: "Rien du tout. " Et Schneiders de commenter: "Je n'avais aucune idée de ce que pouvaient être les implications herméneutiques de l'inspiration, mais il me paraissait étrange, si la raison même de notre attachement à ce texte tenait au fait qu'il était de quelque façon "la parole de Dieu”, que cette donnée théologique n'ait aucune signification pour l'interprétation de ce texte» (2005, p. 9). 
dont nous disposons sur lui, mais sur la connaturalité, finalement de nature spirituelle, que nous pouvons développer avec la vérité de son existence, telle que les légendes et les "gestes » en lèguent le symbole vif, même si les exégètes sont requis d'en raviver les contrastes et d'en écarter les falsifications. (p. 127)

Il me semble que Gagey rejoint ici l'intuition que j'essayais de suggérer plus haut. Il le fait à partir de préoccupations différentes, inspiré des débats et réflexions qui s'élaborent à partir de la question des relations entre la Bible comme Parole de Dieu et la dogmatique, comme expression rigoureuse de la vérité religieuse à croire. Mais ce point de départ différent le ramène au même constat: il importe de reconnaître l'objectif spirituel, et même l'approche spirituelle, d'une lecture de la Bible, y compris le Nouveau Testament dans ses propos sur Jésus, pour que le texte devienne vraiment parole de Dieu. Quand je parle d'objectif et d'approche, je veux dire que la dimension spirituelle ne vient pas seulement dans le fruit attendu au terme de la lecture, mais qu'elle doit être présente tout au long de la recherche pour assurer qu'aucun élément significatif n'est écarté en cours de route.

\section{L'expérience spirituelle, lieu de découverte de Jésus. Le cas Marcel Légaut}

Les discussions autour des relations entre le Jésus historique, le Jésus réel et le Jésus actuel (Schneiders 1995), manifestent au moins deux choses. Du point de vue de l'histoire, les critères évoluent à mesure que les méthodes historiques se déplacent et que les informations nouvelles apparaissent. Du point de vue de la théologie, l'importance reconnue à l'histoire varie selon les approches théologiques. Et tout cela est fort louable. Quand Gagey parle de légendes et de gestes, il montre par ailleurs que les résultats obtenus par la démarche critique continuent d'offrir l'occasion de construire des récits tout aussi importants que les affirmations critiques pour l'approche de la personne de Jésus. Cette connaturalité, il l'affirme être de «nature spirituelle».

La question se pose alors de savoir si on ne pourrait pas se tourner franchement du côté de l'expérience spirituelle pour prolonger autrement la réflexion sur le Jésus historique. Si déjà le portrait de Jésus offert dans les écrits du Nouveau Testament est davantage le reflet de l'option des auteurs de ces textes que le portrait exact des faits et gestes de Jésus, ne peut-on pas dire que c'est leur expérience spirituelle qui leur a dicté les traits à reconnaître à Jésus, même si on maintient le consensus actuel sur 
le fait que ces traits entretiennent aussi un lien avec le Jésus qui a réellement existé et qui a été connu de quelques-uns de ces auteurs?

C'est cette hypothèse à mon avis qui soutient la réflexion de Marcel Légaut $^{3}$ et qui lui permet de présenter son portrait de Jésus, tout en disant que ce portrait n'est pas que sa création. Autrement dit et pour faire bref, Légaut affirme qu'en communiant, à partir de son expérience spirituelle, à l'expérience des auteurs des textes du Nouveau Testament et des personnes constituant les premières communautés chrétiennes, il rejoint à sa manière le Jésus de l'histoire, dont il fait le Jésus réel de sa démarche de foi. S'il ne fait pas un travail d'historien et si sa réflexion s'enrichit du progrès des découvertes historiques, le sens qu'il dévoile à partir de son cheminement spirituel est plus important et significatif que les détails dégagés de l'examen critique.

C'est cette position que je voudrais illustrer à partir du livre: Introduction à l'intelligence du passé et de l'avenir du christianisme (1970). Auparavant, il ne sera peut-être pas inutile de présenter rapidement Marcel Légaut.

\section{Un portrait circonstancié}

Légaut est pratiquement contemporain de la deuxième étape de la question du Jésus historique. Né en 1900 à Paris, il sera fortement influencé à l'École normale supérieure de la rue d'Ulm par Monsieur Portal, lui-même une victime de la crise moderniste en raison de son association au mouvement œcuménique naissant. Portal conseillera à Légaut la méditation des évangiles et contribuera ainsi à la naissance d'un réseau dans le monde de l'éducation française.

Un groupe se réunira régulièrement autour de quelques normaliens pour méditer les évangiles et faire circuler le fruit de ses méditations auprès des enseignants catholiques. Durant l'entre-deux-guerres, ce mouvement sera en croissance permanente et il amènera ces jeunes à passer ensemble une partie de leurs vacances d'été pour approfondir leur foi avec des guides comme Teilhard de Chardin et Édouard LeRoy, entre autres.

Après la débâcle française de la Deuxième Guerre mondiale, Légaut s'installera dans la Drôme avec le projet d'élever des moutons et de continuer sa carrière de professeur de mathématiques. Ce projet s'avérera trop

3. J'évoquerai rapidement plus bas qui est Marcel Légaut, mais à qui voudrait une description un peu plus complète, je signale la présentation que j'ai faite de lui dans Breton (2000). 
exigeant et finalement les moutons l'emporteront sur les étudiants: Légaut sera berger jusqu'à sa retraite au début des années 1970.

Il aura toutefois, pendant cette période, repris contact avec les camarades d'avant la guerre et, avec l'aide de certains, il publiera des œuvres majeures sur son expérience spirituelle. Cette longue pratique de méditation des évangiles, seul et en groupe, avait convaincu Légaut de la place unique de Jésus dans son expérience. Mais sa découverte de Jésus était pour ainsi dire à la mesure de son propre approfondissement de son humanité. Pour paraphraser ses propos, je dirais que plus j'entre dans l'intelligence de ce que je suis comme personne humaine, plus j'entre dans la compréhension de Jésus. Plus je m'approche de mon mystère, plus je fais l'expérience du mystère de Jésus. C'est dans ce sens que les deux ouvrages majeurs de Légaut sont, à mon avis et celui de plusieurs autres, L'homme à la recherche de son humanité et Devenir soi.

J'emprunterai ici à un autre ouvrage qui devait, à l'origine, être la suite de L'homme à la recherche de son humanité, mais qui sera de fait publié avant. Dans ce livre, Introduction à l'intelligence du passé et de l'avenir du christianisme, nous allons voir comment Légaut fait l'approche de Jésus à partir de son expérience spirituelle. On sera du coup à même de constater comment l'expérience spirituelle pour Légaut intègre toutes les composantes de la vie d'une personne dans une recherche d'unification toujours en progrès.

\section{Une expérience familière}

Pour illustrer la connaturalité qui entretient les légendes et les "gestes", mais qui vit aussi d'elles, Gagey offre l'exemple de ce qui peut arriver à des personnages historiques, comme Jules César, Napoléon, Gandhi ou François d'Assise. Plus près de nous, on peut penser aux développements que permettraient certains avis de décès ou aux témoignages qui sont offerts à l'occasion de funérailles.

Pas besoin d'être spécialiste de l'histoire pour deviner que ces deux genres littéraires contemporains pourront engendrer des récits, légendes et "gestes » où se mêlent réalité et interprétation. On peut comprendre dès lors le travail qui attend les futurs interprètes et, par analogie, deviner ce qui attend les biblistes, historiens et croyants dans leur lecture des évangiles. La référence à des pratiques contemporaines permet surtout de voir comment dans un récit à portée historique la qualité des informations offertes et la façon de les lire et de les recevoir se conjuguent de différentes manières pour construire des sens différents, opposés ou complémentaires. 
Il est donc permis de dire qu'à notre époque, riche en informations précises, un événement comme la mort est susceptible de donner prise à des récits diversifiés. À une époque comme celle de Jésus, où les événements étaient souvent coulés dans des genres littéraires établis, le souci du détail historique répondait à d'autres attentes que les nôtres. Malgré tous les efforts consentis par les historiens les plus critiques et les biblistes les mieux informés, chaque résultat mis de l'avant au cours des derniers siècles, chaque interprétation proposée, n'en continue pas moins de soulever des questions. Si cela est prévisible et en quelque sorte inévitable, on comprend que le croyant, disciple de Jésus, ait le goût de se construire une image de son maître qui réponde d'abord à ses besoins spirituels. Légaut est un témoin important de cette démarche, même s’il avoue lui-même ne pas proposer une méthode infaillible.

\section{Sans méthode reconnue et garantie}

Dès le départ de sa réflexion, il se doit de l'avouer et de proposer, à partir de son expérience d'enseignant, une approche susceptible de porter fruit en raison de l'engagement de celui qui la pratique plutôt que pour des références méthodologiques précises. Dans son vocabulaire et à partir de son expérience d'enseignant, il dira que l'enseignant dépasse les règles de sa fonction pour accéder aux exigences internes de sa mission.

Il n'est pas de techniques pour réduire et faire disparaitre la distorsion entre fonction et mission. La vertu et le talent n'y suffisent pas. Tout au contraire, la perfection même des méthodes employées par le bon professeur qui n'est cependant que cela, quand il traite de la vie spirituelle, voire de la théologie, donne le change en rendant apparemment clair et évident ce qu'il enseigne. Il empêche ses auditeurs de soupçonner l'épaisseur questionnante mais aussi appelante de ces matières singulières qui, à vrai dire, ne supportent que le témoignage, car hors de la vie de celui qui les expose, elles ne sont plus que l'ombre caricaturale d'elles-mêmes. Le bon professeur, qui n'est pas aussi en quête de ce qu'il enseigne et en lutte continuelle avec son savoir, instruit mais ne pousse pas à la recherche, sans laquelle la foi ne peut connaître un réel épanouissement et reste paralysée, voire asphyxiée dans la gangue des doctrines. Il rassure et assoit dans la conviction celui qui aurait besoin qu'on l'aide à se lever et à partir sous l'exigence de sa foi, sur le chemin qui est le sien, à la rencontre de son être et à la découverte de Jésus. (1970, 77)

Parce qu'il est lui-même en recherche, le bon professeur va permettre à ses étudiants de découvrir un monde derrière les informations qu'il va 
leur communiquer. Bien plus, il les rendra capables de soupçonner des questions auxquelles ils étaient auparavant tout à fait insensibles. Dans sa manière d'être en profondeur, l'enseignant devient un maître plus par ce qu'il est que dans ses paroles. Sa mission s'affiche par ce qu'il devient et elle se réalise à mesure qu'il progresse et grandit. Par la référence accordée au rôle de l'enseignant et de l'étudiant, Légaut se rapproche de ce que des spécialistes nomment l'herméneutique; mais il renonce à ce mot pour ne pas donner le change et faire croire que le processus qu'il évoque pourrait s'enseigner.

Ce qui vaut, chez Légaut, pour l'initiation et la formation spirituelles en général s'applique évidemment avec la même rigueur dans la place reconnue à Jésus pour un croyant de foi chrétienne. Une formation précise à même les meilleures connaissances disponibles lui sera indispensable pour situer ce personnage ancien qu'est Jésus dans son contexte historique. Mais pour entrer en communication profonde avec lui, une autre attitude s'avérera encore plus décisive. Il devra apprendre à méditer, c'est-à-dire à faire advenir dans son présent le passé éloigné, et surtout à en reconnaître le sens pour sa propre vie spirituelle.

En ce siècle, si le croyant est suffisamment approfondi humainement et spirituellement, s'il est capable de la conduire avec recueillement et piété, une telle méditation sur le passé chrétien est sans doute plus importante pour lui que la connaissance, incontestablement utile, des millénaires qui ont précédé et dans une certaine mesure préparé l'avènement de Jésus. Menée de façon assidue le long de sa vie, sans cesse enrichie par l'expérience quotidienne, elle lui apprendra plus que la lecture des Écritures; en effet cellesci ne rapportent que la préparation lointaine et balbutiante à la mission de Jésus, ou n'en sont qu'une première prise de conscience faite par des hommes qui, malgré l'influence directe de Jésus, étaient dépassés par l'événement. Inversement une telle recherche illuminera ces testaments du passé. Sans elle on ne saurait entrevoir l'universel véritable que ces écrits comportent [...] $(1970,86)$

Le croyant fera l'expérience de découvrir un sens nouveau, pour ne pas dire un surplus de sens, à partir de la compréhension de sa propre vie. Plus il entrera dans la compréhension de son propre mystère, plus il découvrira de sens dans les récits évangéliques et dans le personnage de Jésus. Non pas qu'il en devienne capable de résoudre certaines énigmes historiques. Il comprendra plutôt qu'en plus de ces questions, les récits réveillent en lui un écho qui lui donne à comprendre ce que l'histoire seule est impuissante à dégager. 
Grâce à sa réflexion et à son expérience de la vie spirituelle, le chrétien, après avoir décanté le passé, le ressuscitera vraiment en lui à partir du peu qu'il en sait; il le comprendra du dedans à la mesure de sa propre maturité. Sans doute fera-t-il cette recherche à ses risques et périls. Il ne peut en être autrement [...] À la lumière de son expérience spirituelle, il s'efforcera de faire le départ entre la fin qui était recherchée et l'esprit fondamental du message de Jésus tel qu'il peut l'entrevoir. Il réfléchira sur les résultats positifs obtenus mais aussi et surtout sur les complicités acceptées, sur les abâtardissements favorisés sciemment ou non, finalement sur les déformations spirituelles qui ont été ainsi provoquées. Il ne se refusera pas à considérer dans leur gravité les tares de la pratique religieuse et leurs conséquences, qui ont pesé sur le développement humain des chrétiens et souvent l'ont retardé et gauchi. Il ne sousestimera pas non plus l'ampleur de la déchristianisation qui en est la conséquence, celle-ci s'expliquerait-elle en partie par d'autres raisons. $(1970,86-87)$

Comme l'enseignant accomplit sa mission en exerçant sa profession à partir de ce qu'il devient, le disciple de Jésus grandit dans sa vision de son maître dans un processus de maturité. Il importe ici de souligner la référence à la maturité spirituelle que le croyant acquiert à mesure qu'il vit et qu'il apprend de ce qu'il vit. Cette maturité peut amener à remettre en question des positions tenues auparavant comme certaines et indiscutables, mais qui apparaissent tout à coup intenables à la lumière de l'expérience acquise. Comment ne pas penser ici à la foi nue de Jean de la Croix ? Légaut préférera utiliser une expression qui a toutes les apparences d'un pléonasme, mais qui dit bien la conscience de tenir ses convictions de foi à la seule force de sa foi. Il parlera «du croyant de foi ». D'un autre point de vue, on peut penser à toutes ces craintes que les croyants éprouvent parfois face à la nécessité d'abandonner les convictions anciennes pour accepter de grandir dans la foi. Il y a là des appels auxquels il n'est pas permis de renoncer sans mettre en danger son progrès spirituel.

\section{L'exigence des appels personnels: la mission}

Nous avons déjà utilisé le mot mission en évoquant le sens particulier que lui reconnaît Légaut. Pour lui, la mission ne renvoie évidemment pas au travail missionnaire à l'étranger et auprès des «païens ». Elle n'est même pas un travail mené dans sa communauté d'appartenance pour consolider ou faire grandir cette communauté. Non, la mission dont parle Légaut se rapprocherait plutôt de ce que l'on a traditionnellement nommé la vocation. Sans doute parce que le mot vocation a pris dans l'histoire une conno- 
tation cléricale et religieuse, Légaut préfère parler de mission pour dire l'appel particulier que chaque personne doit découvrir et auquel elle doit répondre de son mieux pour se réaliser. Pour entendre les appels qui permettront d'entrevoir sa mission, il faudra renoncer à certaines distractions et développer une capacité d'écoute particulière. Ce sera particulièrement le cas dans la découverte de qui est Jésus pour cette personne.

Tant que l'homme demeure dans l'euphorie de son action et dans la ferveur de ses croyances, qu'elles soient religieuses, sociales ou politiques, trop absorbé par ce qu'il fait, trop assuré de ses doctrines, il ne saurait s'intéresser réellement aux questions qui se posent à l'occasion de Jésus. Cette recherche ne peut que lui paraître accessoire, relever d'un savoir quelconque ou même d'une simple curiosité, d'autant plus qu'elle concerne un passé déjà lointain, dont les conséquences s'amenuisent visiblement et sont condamnées sans doute à disparaître à la longue, malgré leur importance encore non négligeable [...] $(1970,11)$

Il arrive aussi que l'on se refuse aux initiatives originales que cette recherche nécessite en se concentrant exclusivement dans l'exercice de méthodes strictement objectives. Cette détermination est commandée par la conviction, maîtresse de l'opinion, véritable croyance, que la science épuise le réel ou encore que rien ne vaut en dehors de ce que celle-ci apporte. [...] À partir de ces données il ne veut faire aucune place dans ce travail à l'activité spirituelle qui permet d'entrevoir un être dans son irréductible particularité. $(1970,11)$

En exergue à un autre de ses livres, Mutation de l'Église et conversion personnelle, Légaut a écrit: "L'Église, ma mère et ma croix". Il voulait, par ces mots, dire sa reconnaissance à l'Église qui lui a permis de naître à la foi, mais aussi les obstacles qu'elle sème parfois sur son chemin de croyant. Dans l'extrait cité ici, Légaut redit autrement la même chose, sans parler de l'Église, mais en évoquant les situations objectives et les croyances convaincues qui permettent à un croyant de naître et de se mettre en marche, mais qui le soustraient éventuellement à l'épreuve de devenir luimême.

Un point fort de la démarche spirituelle est la reconnaissance de cette particularité irréductible de chaque personne. Parce que chacun est différent, parce que chacune est singulière, la voie spirituelle varie avec chaque individu, comme l'expérience de la rencontre de Jésus. Jésus, en effet, cesse alors d'être un objet d'étude ou même le but identifié d'une quête: il devient lui plutôt un sujet unique dans la relation qui se construit avec celui ou celle qui cherche à le rencontrer. 
En vérité, de même que l'homme se trabit en ne voulant pas se connaître comme sujet, il renonce à la connaissance d'autrui en ne faisant de celui-ci qu'un objet. Nul ne peut être atteint réellement ainsi, Jésus moins que personne à cause de sa taille exceptionnelle. Une connaissance scientifique de Jésus, même plus complète et plus poussée dans le détail qu'elle n'est possible en fait, resterait encore illusoire. L'histoire de Jésus si courte, aux conséquences si démesurées qu'elles paraissent invraisemblables, va jusqu'à le rendre assez difficile à concevoir pour qu'on ait été conduit, avec une intrépidité qui n'allait pas, certes, sans témérité, jusqu'à douter de son existence. $(1970,12)$

Ce que nous expérimentons déjà dans les relations humaines qui ne se contentent pas d'être de simples contacts épidermiques se reproduit à une échelle infinie dans le cas de Jésus. Plus nous avons appris à découvrir en l'autre davantage qu'un simple objet de rencontre, plus nous avons découvert et expérimenté la richesse d'une relation profonde, plus Jésus peut nous apparaître dans la profondeur accordée à notre capacité d'accueil. Pour parodier les titres d'ouvrages de Légaut, plus l'homme progresse dans la recherche de son humanité, plus il devient soi, plus Jésus prend un sens inédit pour lui. Cette relation unique et originale est d'une certaine manière illustrée par les propos de Légaut sur la différence entre la religion d'autorité et la religion d'appel. Quand Jésus s'inscrit dans une relation spirituelle unique avec le croyant, les exigences ne s'imposent plus au nom d'une religion qui dicte des règles avec autorité, mais au nom de la nécessité inscrite dans les appels entendus.

Dans cette autre religion, connaître Jésus, c'est le chercher plus que le définir à partir d'une théologie qui satisfait l'intelligence ou du moins lui donne quelque pâture; correspondre à Jésus c'est s'efforcer à se pénétrer de son esprit plus qu'à se conformer et à obéir scrupuleusement à la lettre de ce qu'il a dit ou commandé de son temps dans des conditions tout autres; c'est viser à lui ressembler autant que cela est possible sans nécessairement l'imiter. [...] Cette autre religion appelle ses fidèles à la progressive intelligence de ce que Jésus a vécu intimement de son temps et ne se borne pas, contrairement à la précédente, à leur enseigner ce qu'ils sont censés avoir à connaître de lui. $(1970,15)$

"Correspondre à Jésus». On pourrait jongler avec cette formule pour en exploiter toutes les significations et toute la richesse d'évocation, mais de la dire simplement suffit pour montrer que, dans son expérience spirituelle, le croyant s'accorde, comme les musiciens de l'orchestre, à ce qu'il découvre de Jésus, sans pour autant disparaître dans un quelconque élan 
fusionnel. Légaut évoque ici de façon implicite tout ce qui peut faire obstacle à une religion d'appel. Contrairement à la religion d'autorité, celle-ci n'assure pas l'identité avec certitude; elle n'indique pas objectivement et pour tous les voies à suivre et elle n'offre pas de critères assurés d'authenticité. Par contre, l'accord, la correspondance de la religion d'appel, où se conjuguent l'offre de Jésus et la quête du croyant, vient en quelque sorte combler ce que la connaissance ne pourra jamais apporter.

À vrai dire, il n'est pas possible de connaître Jésus de Nazareth tel qu'on pouvait le voir et l'entendre quand il parlait et agissait. Il n'a rien écrit. Son action auprès des hommes n'a connu une extension importante que pendant quelques mois. On ne peut l'atteindre qu'à travers ce que ses disciples ont vu de lui, ont retenu et compris de ses actes et de ses paroles. Les faits de sa vie, ses actions comme ses discours, ne sont connus que par une tradition orale; cette tradition n'est devenue écrite qu'à une époque relativement tardive; fervente sans nul doute, fidèle d'intention certes. Mais par l'intérêt qu'elle soulevait, était-elle pourtant à l'abri des commentaires qu'elle appelait, des additions qui l'enjolivaient et qui lui convenaient trop bien, semblait-il, pour n'être pas amalgamées avec elle? [...] Il est impossible d'apprécier le degré d'exactitude des textes qui transmettent cette tradition issue des origines chrétiennes [...] $(1970,16)$

Il y a un deuil à vivre eu égard aux attentes cognitives à propos du Jésus historique et cela, non seulement parce qu'il y aura toujours des informations manquantes ou insuffisantes, mais parce qu'il s'agit de rencontrer un sujet en tant que tel. Faut-il pour autant bouder les informations disponibles et refuser d'en chercher de meilleures et de plus précises?

\section{Sans refuser la science}

Tout ce qui précède ne doit toutefois laisser croire que Légaut était indifférent ou opposé aux informations scientifiques. Mais avant de faire état de ses positions à la fin de sa vie, commençons par bien situer les opinions exprimées dans le livre que nous utilisons ici.

Cependant, ce que les hommes peuvent connaître objectivement de Jésus à partir des traditions orales et écrites de ses disciples est capital, même si ces données sont absolument insuffisantes pour faire franchir le seuil qui ouvre sur son mystère. Les progrès réalisés par les sciences historiques et l'exégèse dans les temps modernes permettent d'affirmer qu'au $\mathrm{Xx}^{\mathrm{e}}$ siècle on sait un peu mieux ce qui s'est passé au début de l'ère chrétienne. Mais ces connaissances nouvelles n'ont guère contribué qu'à dégager la figure de Jésus de ce 
qu'y avaient ajouté les idéologies fabulatrices, l'imagination et la dévotion des fidèles. Non sans quelque ironie on peut même penser, qu'en dehors de questions de détail, les résultats de portée générale déjà obtenus, fruit d'un travail immense et minutieux, relèvent principalement du bon sens lorsqu'il est assez nourri par l'expérience de la vie et dégagé de tout a priori systématique. Les chrétiens, par leurs initiatives, et non, comme ce fut presque toujours le cas, en se traînant derrière les non-chrétiens, auraient dû depuis longtemps promouvoir ces progrès que, en droit, leur foi appelle pour être plus purement elle-même. Sans ce lourd appareillage technique, ils auraient dû les réaliser grâce à leur intelligence religieuse si elle avait été vigoureuse et délivrée de toute entrave intérieure ou extérieure. $(1970,17)$

Voilà qui n'est pas très élogieux à l'égard des connaissances fournies par les biblistes. Bien sûr, Légaut reconnaît la situation nouvelle de son $\mathrm{xx}^{\mathrm{e}}$ siècle, après tout il est contemporain de la crise moderniste, mais il réduit les résultats de la science au gros bon sens. Un peu comme si les connaissances acquises récemment n'avaient fait que fournir un nouveau répertoire d' «idéologies fabulatrices». Il serait d'ailleurs assez facile de trouver d'autres citations qui élargissent cette appréciation à tout le champ de la théologie. Pour une longue période de sa vie, Légaut a gardé une méfiance certaine à l'égard des savoirs et des savants qui prétendaient encadrer l'expérience spirituelle et parler de Dieu comme d'un objet habituel du savoir.

Même à cette période de sa vie où il était critique à l'égard de l'exégèse et de la théologie, il était encore capable toutefois d'anticiper le profit possible de connaissances plus justes et précises. Il avait déjà l'intuition de cette situation dans des propos comme ceux qui suivent:

L'homme n'exerce pas seulement son activité spirituelle pour se souvenir de ce qu'il a été, pour être ainsi présent à lui-même et entrer dans son existence. Son intelligence se porte aussi sur la vie et les œuvres de ceux dont il veut s'approcher pour comprendre en profondeur qui ils sont et se les rendre présents. Quand il n'a pas connu personnellement quelqu'un, sa mémoire ne lui sert de rien mais d'autres éléments peuvent jouer le rôle des matériaux que, dans ces conditions, elle ne saurait procurer: ainsi des lettres, des écrits de cet auteur, des témoignages à son sujet. L'activité que l'homme déploie sur cet ensemble de données pour entrer dans le mystère de l'autre ressemble, en tout point à celle que, pour se souvenir, il exerce sur les matériaux fournis par sa mémoire. À son initiative et sous sa responsabilité, à la lumière de son expérience personnelle et de son sens de l'humain, il fait le départ dans ce dossier entre ce qui est important et ce qui ne l'est pas [...] Il entrevoit ainsi progressivement la signification fondamentale de cette existence. $(1970,19)$ 
Il est instructif de constater comment ce mathématicien conjuguait sa soif de savoir scientifique avec sa quête spirituelle. Il savait faire la part des choses et, surtout, il tenait à ce que tout le savoir fourni par la science soit approprié pour servir à la croissance de l'homme. On pourrait encore illustrer cela par ses propos sur la mémoire et le souvenir (voir Breton 1987, 50-53), mais cela nous entraînerait loin de nos réflexions actuelles. Concluons plutôt sur ce point par une dernière citation où Légaut réaffirme que ses explications ne tiennent pas à des élucubrations abstraites, mais qu'elles répondent pour lui à l'exigence intime de croissance, inscrite au cœur de toute personne. En somme, au-delà des informations historiques, Jésus prend pour chacun le sens que celui-ci s'est préparé à reconnaître et accueillir, comme c'est le cas dans l'expérience quotidienne:

La maturité de l'homme donne leur mesure à la justesse et à la profondeur de sa vision de l'autre, à l'exactitude des jugements qu'il porte sur lui, à la plénitude de la synthèse, d'ailleurs sans cesse à reprendre, qu'il fait à son sujet. Cette intelligence active varie avec son état spirituel, grandit quand il s'approfondit lui-même, et aussi se défait avec lui. Nul n'est plus capable de découvrir l'esprit fondamental d'un autre que celui qui a déjà quelque sens du sien propre $[\ldots](1970,20)$

Légaut ira encore plus loin dans son appréciation de la science et de son apport à la croissance spirituelle. À ce sujet, je voudrais partager une expérience vécue. Quelques années avant sa mort, j'étais, avec un groupe d'amis de Légaut, à faire avec lui la relecture de ses ouvrages. Pendant une pause, il m'avoua en toute honnêteté et en parlant de ses écrits des années 1970 et 1980: «Ce que je pouvais être ignorant à cette époque!». De même qu'il avait profité, au début de sa vie spirituelle, de maîtres comme Portal, Teilhard de Chardin et Édouard LeRoy, sans oublier les compagnons comme Gabriel Marcel, il s'est instruit, dans ses dernières années, de la présence de certains biblistes qui fréquentaient Mirmande 4 et qui lui permettaient d'élargir ses connaissances. Il était très content de pouvoir

4. Au cours des années 1970, les amis de Marcel Légaut ont acheté avec lui une magnanerie à Mirmande dans la Drôme. Au cours de l'été, des groupes se succédaient ainsi pour faire connaissance de la pensée de Légaut ou l'approfondir en sa compagnie et celle des plus anciens. Depuis la mort de Légaut en 1990, la maison est devenue la propriété de l'Association Marcel-Légaut et elle continue d'accueillir des groupes qui s'intéressent à Légaut et à quelques autres comme Jean Sullivan ou Olivier Rabut, par exemple. De même, à l'occasion de ses passages au Québec de 1979 à 1981, Légaut a particulièrement apprécié les rencontres organisées avec des théologiens. 
ainsi s'instruire, même si cela ne remettait pas en question ses convictions sur l'expérience spirituelle.

Comme je viens de le dire, le Légaut des dernières années était devenu plus respectueux du résultat des démarches exégétiques. Il aurait sans doute accepté que ces recherches puissent faire plus que simplement mettre à l'abri des erreurs grossières et de l'ignorance crasse. Et il était même parvenu à apprécier ce que les nouvelles méthodes apportaient de suggestif et d'intelligent à la lecture de la Bible, surtout du Nouveau Testament pour lequel il gardait une préférence. Ce changement, qu'on pourrait peut-être appeler une conversion en douceur, s'explique à mon avis en raison des conditions nouvelles dans lesquelles il réfléchissait à la fin $\mathrm{du} \mathrm{xx}^{\mathrm{e}}$ siècle. Son initiation à la méditation de la Bible inaugurée dans la foulée de la condamnation du modernisme s'était effectuée dans un tout autre contexte. Il avait gardé de cette époque une certaine méfiance pour les personnes, biblistes ou théologiens, qui s'enfermaient dans un langage objectif, dicté en même temps par un certain dogmatisme, et qui refusaient ainsi de faire une juste place au mouvement de foi. À mesure qu'il a rencontré des théologiens et biblistes qui n'étaient pas que des techniciens de leur savoir, il a découvert l'utilité de leurs connaissances. Il anticipait ainsi certains discours que nous entendons aujourd'hui et que je voudrais évoquer rapidement, non pas comme une preuve de la valeur des positions de Légaut, mais comme une illustration différente de la voie qu'il suggérait.

\section{Des échos aux réflexions de Légaut}

J'ai déjà cité plus haut l'ouvrage de Gagey, La vérité s'accomplit; je voudrais maintenant ajouter quelque peu à cette référence. Ensuite, j'évoquerai l'entreprise de Schneiders.

Dans le livre déjà cité, Gagey aborde en premier lieu les conditions de travail de la théologie pratique, comme participante à l'élaboration de la vérité. Puis il passe, dans sa deuxième partie intitulée "La vérité en procès " au conflit entre exégèse et dogmatique, selon son vocabulaire. C'est dans ce contexte qu'il consacre un chapitre au "consensus théologique issu de la "deuxième quête" ".

J'ai déjà cité le passage où il affirme que ce procès aboutit finalement à reconnaître la connaturalité de nature spirituelle. Mais auparavant, il a situé ce procès dans un contexte plus ecclésial.

L'accumulation des informations exactes attendue de la quête historique ne saurait donc dispenser quiconque d'avoir à prendre position dans le procès 
qu'ouvre la lecture du récit fait avec l'autorité qui fut celle de Jésus et qui est désormais répercutée là où le récit est proclamé comme la Parole de Vie qui vient éprouver les figures d'espérance dont vit son auditeur. C'est ce qui se produit là dans l'Église née de Pâques. Sans doute, de ce procès l'historien peut-il éclairer les tenants et aboutissants, mais il peut tout autant empêcher qu'il s'accomplisse en le différant sans cesse quand l'interminable quête du savoir ensevelit l'urgence de l'interpellation. (Gagey 2009, 126)

Gagey limite d'un autre point de vue que Légaut le pouvoir du savoir qu'il reconnaît à l'historien. Si on cède au seul désir de savoir de ce dernier, ne risque-t-on pas en effet de ne jamais revenir au but pour lequel on a commencé à s'intéresser à ce procès: connaître Jésus pour le rencontrer. Comme l'historien ne pourra jamais reconstituer exactement le "Jésus terrestre qui jadis appela à la foi »(Gagey 2009, 129), de même "il est vain d'espérer savoir ce qu'il en est de Jésus en vérité. Cette vérité en effet n'est pas l'objet d'un savoir, mais engagement de liberté. Elle se présente donc comme "une vérité à faire" [...]» (p. 133).

Il n'est pas nécessaire d'insister pour comprendre comment Gagey rejoint en partie l'approche de Légaut. Favorable lui aussi à la démarche historique qui instruit et épargne les erreurs grossières, il maintient, du point de vue de la communauté croyante, la nécessité d'un engagement pour rejoindre Jésus en vérité. Il aurait été intéressant de pousser plus loin un éventuel dialogue entre Légaut et Gagey sur ce sujet. S'ils semblent emprunter des voies différentes en raison de leurs points de départ, l'engagement évoqué renvoie à la démarche spirituelle de l'individu qui s'engage en Église. De son côté, Légaut insistait pour affirmer d'abord la mission individuelle comme lieu de croissance spirituelle, mais l'ensemble de son œuvre a fait une large place à l'apport de la communauté de foi, qui peut se vivre explicitement en Église ou pas, dans le cheminement spirituel. La connaturalité dont parlait Gagey me semble bien illustrée dans l'expérience et les propos de Légaut. Sans déprécier les apports des sciences bibliques, force est de constater que le Jésus qui a vécu à un moment de l'histoire devient quelqu'un pour le croyant ou la croyante d'aujourd'hui en raison et à la mesure de la vie spirituelle de cette personne. Schneiders propose des conclusions similaires à partir de son point de vue de bibliste et d'experte en spiritualité.

Dans Le texte de la rencontre, elle veut explorer toutes les composantes du travail biblique qui s'intègrent à la démarche herméneutique et qui permettent un accès au texte biblique utile à la vie spirituelle. En d'autres mots, elle veut contester l'opinion de son professeur de l'Institut 
catholique de Paris et montrer que l'aboutissement normal des méthodes utilisées par les biblistes ne doit pas s'arrêter au savoir dit scientifique, mais qu'il est mené à des fins spirituelles.

Illustrons cette position à partir de ses explications sur le Jésus historique. Après avoir retracé le contexte historique déjà présenté ici et avoir fourni les explications de sens des mots Jésus réel, historique et proclamé (Schneiders 1995, 170ss), elle met de l'avant ce que j'appellerais une clef de lecture: l'imaginaire pascal, "pour désigner l'imagination théologique et spirituelle chrétienne» (p. 173ss).

Empruntant à des travaux antérieurs sur l'imaginaire constructif, Schneiders les applique à l'événement pascal. Elle reprend d'abord cinq caractéristiques de l'imaginaire constructif. Il est dynamique en ce sens qu'il produit une image unifiée porteuse de sens. Les images produites «se développent et opèrent de façon herméneutique ». À partir du jeu de l'imagination, la personne fait des rapprochements et saisit des aspects jusque-là ignorés. Ensuite, cet imaginaire ne fournit jamais des images "précises et parfaitement claires". Un peu comme dans l'ancien discours sur l'analogie, on se doit ici d'enregistrer des affirmations en les accompagnant de négations, d'où le flou et l'imprécision dans le sens offert. Les images fournies par l'imaginaire constructif sont aussi arrimées aux trois dimensions du temps: le passé, le présent et l'avenir. Il y a là un retour dans le passé à partir du présent qui ouvre une porte sur le futur. Enfin ces images sont "chargées d'affectivité » au sens où elles sont l'occasion de relire l'expérience personnelle de qui les reçoit.

Une fois présenté l'imaginaire constructif, Schneiders l'applique à l'événement pascal dans ce qu'elle appellera dès lors l'imaginaire pascal. L'image de Jésus dessinée par l'Église primitive est «dynamique et globale ", elle donne un sens aux événements vécus comme le raconte le récit des disciples d'Emmaüs. Cette image de Jésus devient du coup herméneutique et s'impose comme principe d'interprétation. Comme en témoigne la multiplicité des récits du Nouveau Testament, cette image de Jésus n'épuise pas la réalité et il y a toujours des aspects qui lui échapperont et qui seront l'objet de nouveaux récits. On comprend aussi que l'image de Jésus s'enracine dans le passé, porte le présent et ouvre sur le futur. «Finalement, l'image de Jésus a été chargée d'affectivité. L'attachement passionné de ses disciples à Jésus a orienté et éclairé leur relation permanente avec lui et les uns avec les autres, comme il a orienté la présentation qui en était faite dans la prédication et l'enseignement » (Schneiders 1995, 181). 
Ces propos sur l'imaginaire pascal offrent une compréhension des récits contenus dans le Nouveau Testament. Ils montrent l'élaboration des récits et, en même temps, ils légitiment la proposition de nouveaux récits actuels, par une nouvelle intervention de l'imaginaire pascal. Comme l'imaginaire pascal a permis aux premiers disciples de donner un sens aux événements vécus avec Jésus autour de sa mort-résurrection, ce même imaginaire invite les croyants d'aujourd'hui à se construire une lecture théologique féconde pour leur expérience spirituelle.

Aux personnes inquiètes qui s'interrogeraient sur la possibilité de construire ainsi un objet de foi offert à l'assentiment de nos contemporains à partir de récits qui ne répondent pas aux exigences scientifiques actuelles, Schneiders répond ceci:

Que le compte rendu évangélique soit substantiellement vrai, cela nous est garanti par le fait que la communauté croyante primitive l'ait accepté, elle dont la foi dépendait autant que la nôtre du témoignage véridique sur l'événement-Christ en Jésus. Autrement dit, la foi fait partie, et doit faire partie, de la question de la fiabilité historique de l'Évangile, non pas comme un substitut à la recherche historique qui doit être poussée aussi loin qu'elle peut, mais comme un élément d'une tradition qui garantit la fiabilité de l'ensemble, quoi qu'il en soit des questions que l'on peut poser ou de la difficulté à expliquer les détails. $(1995,184)$

Sans qu'il soit nécessaire de parler de preuves, même circonstancielles, il est intéressant de voir comment trois démarches différentes arrivent à des conclusions similaires sur les rapports souhaitables entre les recherches historiques et les démarches plus spirituelles dans la compréhension de Jésus. Au lieu d'opposer le Jésus de l'histoire à celui de la foi et d'insinuer que l'importance de l'un diminue à mesure que celle de l'autre augmente, on comprend que les deux se complètent et d'une certaine manière s'alimentent l'un et l'autre. J'ai insisté davantage sur les positions de Légaut, car il est à l'origine de ce que je propose. Mais les références rapides à Gagey et Schneiders suggèrent une convergence qu'il ne faudrait pas oublier quand on évalue les progrès et les retombées des recherches historiques. Car la croissance spirituelle s'avère le plus souvent le facteur déterminant dans l'élaboration d'une relation avec Jésus qui rejoint la mission pour laquelle il est venu parmi nous. 


\section{Références}

Breton, J.-C. (1987), Foi en soi et confiance fondamentale. Dialogue entre Marcel Légaut et Erik H. Erikson, Montréal/Paris, Bellarmin/Cerf (Recherches / Nouvelle série 13). Fides.

Gagey, H.-J. (2009), La vérité s'accomplit, Paris, Bayard (Theologia).

KäsemanN (1970) [1954], "Le problème du Jésus historique », Essais exégétiques, Neufchâtel, Delachaux et Niestle, p. 145-173.

LÉgaut, M. (1970), Introduction à l'intelligence du passé et de l'avenir du christianisme, Paris, Aubier (Intelligence de la foi).

(1971) L'homme à la recherche de son humanité, Paris, Aubier (Intelligence de la foi).

(1975) Mutation de l'Église et conversion personnelle, Paris, Aubier (Intelligence de la foi).

(1980) Devenir soi, Paris, Aubier (Questions spirituelles).

Marguerat, D., E. Norelli et J.-M. Poffet (2003²), dir., Jésus de Nazareth. Nouvelles approches d'une énigme, Genève, Labor et Fides (Le Monde de la Bible 38).

Meier, J. P. (2004-2005), Un certain Juif, Jésus. Les données de l'histoire, 3 vol., Paris, Cerf.

SChNeiders, S. M. (1995), Le texte de la rencontre, Paris/Montréal, Cerf/ Fides (Lectio divina 161).

Schweitzer, A.(1994) [1906], «Histoire des recherches sur la vie de Jésus: considérations finales ", Études théologiques et religieuses, 69/2, p. 153164.

\section{Résumé}

Le présent article formule une opinion selon laquelle la compréhension de Jésus est fortement conditionnée par la vie spirituelle de ceux et celles qui s'intéressent à lui. Sans négliger les résultats de l'histoire, il appert bien souvent que le sens accordé à l'image de Jésus retenue dépend plus de la vie de foi et des engagements personnels que de la force des arguments scientifiques. Le Jésus réel échappera toujours en partie à la quête historique, mais il peut s'imposer aux personnes qui le découvrent avec les yeux de leur foi. 


\section{Abstract}

This paper argues that the understanding of Jesus highly depends on the spiritual life of those who seek him out. Even when one accepts the results of historical investigations, it often appears that the meaning given to Jesus' image one holds comes more from one's faith and one's personal commitment than from the strength of scientific evidence. The real Jesus will always evade the historical quest, but he may manifest himself to those who choose see him with their faith. 